\title{
QUALIDADE DE VIDA EM PORTADORES DE ESCLEROSE MÚLTIPLA
}

\author{
Rogério de Rizo Morales', Nívea de Macedo Oliveira Morales², \\ Fernando Coronetti Gomes da Rocha ${ }^{3}$, Sheila Bernardino Fenelon 4 , \\ Rogério de Melo Costa Pinto ${ }^{5}$, Carlos Henrique Martins da Silva ${ }^{6}$
}

\begin{abstract}
RESUMO - A esclerose múltipla (EM) é doença crônica que pode causar repercussões importantes na vida dos pacientes. Medidas tradicionais de evolução na EM não consideram os efeitos da doença na qualidade de vida relacionada à saúde (QVRS). O objetivo deste estudo é avaliar o impacto da EM na QVRS de portadores residentes em Uberlândia - MG. O SF-36 foi aplicado em 23 portadores de EM e em 69 doadores de sangue. Os portadores de EM apresentaram escores mais baixos que a população geral, principalmente nos domínios de função física. Pacientes com EDSS $\leq 3,5$ apresentam escores maiores em quatro domínios do SF-36 que aqueles com EDSS $\geq 4,0$, e menores em todos os domínios em relação ao grupo controle. Sintomas depressivos e intolerância ao calor mostram correlação com domínios e componentes do SF36. Concluindo, a EM provoca impacto negativo significante em todos os domínios do SF-36, comparados à população geral, mesmo nas fases de menor incapacidade.
\end{abstract}

PALAVRAS-CHAVE: esclerose múltipla, qualidade de vida, avaliação em saúde.

\begin{abstract}
Health-related quality of life in multiple sclerosis
ABSTRACT - Multiple sclerosis (MS) is a chronic disease which may exert significant effects on the life of patients. Traditional outcome measures in MS lack in consider the effects of the disease on health-related quality of life (HRQoL). The goal of this study is to measure HRQoL in MS patients in the city of Uberlândia, State of Minas Gerais, Brazil. The Brazilian version of the SF-36 was applied in 23 MS patients and in 69 subjects of general population (blood donors) in Uberlândia. MS patients scored lower in all SF-36 scales than do the general population, principally in physical function domains. Patients with EDSS scores $\leq 3.5$ had higher mean scores in four domains than do the patients with EDSS scores $\geq 4.0$, and lower in all domains than control group. Depressive symptoms and heat intolerance showed correlation with SF-36 domains and components. In conclusion, MS patients have a significant negative impact on all HRQoL domains measured by SF-36, compared with general population, even in the stages with lower disability.
\end{abstract}

KEY WORDS: multiple sclerosis, quality of life, health evaluation.

A esclerose múltipla (EM) é doença crônica, de caráter inflamatório e degenerativo ${ }^{1}$, que acomete preferencialmente adultos jovens ${ }^{1,2}$. A saúde e o bem estar desses indivíduos podem sofrer forte impacto pela doença e/ou efeitos colaterais de medicamentos, com interferência significativa na qualidade de vida (QV) de seus portadores. O conceito de QV refere-se a indicadores objetivos e subjetivos de felicidade e de satisfação ${ }^{3}$. Segundo a Organização Mundial de Saúde, a QV é definida como "a percepção do in- divíduo de sua posição na vida, no contexto cultural e no sistema de valores em que ele vive e em relação a seus objetivos, expectativas, preocupações e desejos" ${ }^{4}$. Na área biomédica, a QV relacionada à saúde (QVRS) refere-se à satisfação e bem-estar do indivíduo nos domínios físico, psicológico, social, econômico e espiritual em relação ao estado de saúde, uma combinação do estado de saúde com a resposta afetiva a esta condição ${ }^{5}$. Entre os instrumentos de avaliação da QVRS, destaca-se o Medical Outcomes Stu-

Propedêutica Neurológica, Faculdade de Medicina, Universidade Federal de Uberlândia, Uberlândia MG, Brasil (UFU): ${ }^{1}$ Mestre em Ciências da Saúde; Médico Neurologista da UFU, Médico do CETEM (Centro de Estudos Triangulino em Esclerose Múltipla) da UF, Membro do BCTRIMS (Comitê Brasileiro de Tratamento e Pesquisa em Esclerose Múltipla); ${ }^{2}$ Mestre em Ciências da Saúde, Médica Neuropediatra da UFU, Membro do BCTRIMS; ${ }^{3}$ Doutor em Neurologia, Professor Assistente Doutor da Faculdade de Medicina de Botucatu - UNESP, Botucatu SP, Brasil, Membro do BCTRIMS; ${ }^{4}$ Doutora, Professora Adjunta IV de Neurologia da UFU; Coordenadora do CETEM, Membro do BCTRIMS; ${ }^{5}$ Doutor, Professor Adjunto III de Estatística da UFU; ${ }^{6}$ Doutor, Professor Adjunto do Departamento de Pediatria da Faculdade de Medicina da UFU.

Recebido 22 Setembro 2006, recebido na forma final 14 Dezembro 2006. Aceito 15 Fevereiro 2007. 
$d y$ - 36-item Short Form (SF-36) ${ }^{6}$, traduzido para o português e validado para a população brasileira ${ }^{7}$. $O$ SF-36 é um instrumento genérico multidimensional, válido e confiável no estudo da QVRS em portadores de $\mathrm{EM}^{8-10}$, mas ainda não utilizado em pacientes brasileiros. Em nosso meio, o primeiro instrumento utilizado para esse fim foi a Escala de Determinação Funcional da Qualidade de Vida na Esclerose Múltipla (DEFU) ${ }^{11}$, versão traduzida e validada para a população brasileira do instrumento específico Functional Assessment in Multiple Sclerosis (FAMS) ${ }^{12}$.

A utilização de um instrumento genérico confiável e válido é necessária para se caracterizar melhor o impacto da EM na QVRS de portadores brasileiros. O conhecimento desse impacto poderá fornecer informações valiosas para pesquisa, além de orientar e aprimorar estratégias de acompanhamento e tratamento oferecidas pelos serviços de saúde em nosso país.

Este estudo tem como obejtivo determinar o impacto da esclerose múltipla na qualidade de vida relacionada à saúde de seus portadores por meio da versão brasileira de um instrumento genérico, o Medical Outcomes Study 36-item Short Form Health Survey Questionnaire (SF-36), em relação à população saudável, e segundo o grau de incapacidade física determinado pelo Expanded Disability Status Scale (EDSS).

\section{MÉTODO}

População - Trata-se de um estudo transversal, aprovado pelo Comitê de Ética em Pesquisa da Universidade Federal de Uberlândia (UFU) e realizado entre janeiro e juIho de 2004. Foram convidados a participar pacientes com diagnóstico de EM de acordo com os critérios do Painel Internacional $^{13}$, atendidos no ambulatório do Centro de Estudos Triangulinos em Esclerose Múltipla (CETEM) da UFU e em clínicas particulares de neurologia e neurocirurgia do município de Uberlândia. Foram excluídos pacientes com incapacidade de compreensão e preenchimento do questionário e portadores com surtos relatados nos últimos três meses antes da entrevista.

Para o grupo controle, foram recrutados doadores voluntários de sangue do Hemocentro da UFU, não portadores de doenças crônicas, pareados com o grupo de pacientes por sexo, na proporção de três controles para cada portador.

Procedimento - Após o consentimento livre e esclarecido, foi realizada uma entrevista para a aplicação do protocolo previamente estabelecido pelos autores, que inclui dados sobre o indivíduo (dados demográficos e profissionais), família (possíveis problemas de saúde e auxílio na renda familiar) e, no grupo de portadores, sobre a doença (história natural, co-morbidades, sintomas e medicamen- tos), além do questionário SF-36, por examinadores treinados. O comprometimento funcional dos portadores foi avaliado por neurologista clínico durante a entrevista, de acordo com o EDSS.

Os pacientes foram divididos em dois grupos, conforme o nível de comprometimento funcional:

- pacientes sem incapacidade para deambular - EDSS entre 0 e 3,5;

- pacientes com alguma incapacidade ambulatorial EDSS igual ou maior que 4,0.

\section{Instrumentos}

The 36-item Short Form Health Survey Questionnaire (SF-36) - O SF-36 é um instrumento genérico para a avaliação de QVRS, amplamente utilizado em diversas condições de saúde, traduzido para o português, adaptado culturalmente e validado para a população brasileira ${ }^{9}$, e com propriedades psicométricas adequadas para utilização em portadores brasileiros de $\mathrm{EM}^{14}$.

Baseado em um modelo multidimensional de saúde, o SF-36 divide-se em dois componentes - o físico e o mental. O componente físico é formado pelos domínios capacidade funcional (com dez itens ou questões), estado geral da saúde (com cinco itens), dor (com dois itens) e aspectos físicos (com quatro itens). O componente mental é constituído dos domínios saúde mental (com cinco itens), vitalidade (com quatro itens), aspectos sociais (com dois itens) e aspectos emocionais (com três itens). O item alteração em um ano é computado à parte, não sendo incluído em nenhum dos componentes.

A avaliação de cada item é feita utilizando o método dos pontos somados (método de Likert), com valores que variam de 0 a 100 pontos, sendo que os maiores escores indicam melhor qualidade de vida. Os escores dos domínios são obtidos a partir das pontuações dos itens de cada domínio, assim como os escores dos componentes derivam dos domínios a eles relacionados ${ }^{6}$.

Expanded Disability Status Scale (EDSS) - O EDSS ${ }^{15}$ é o instrumento mais utilizado em ensaios clínicos, estudos de história natural e na prática clínica como medida de incapacidade neurológica na EM. É constituído de duas partes: uma escala formada por nove sistemas funcionais (piramidal, cerebelar, tronco encefálico, sensitiva, vesical, intestinal, visual, mental e outras) e uma escala principal - o EDSS propriamente dito - ordinal, não contínuo, com valores de 0 a 10 e com incrementos de 0,5 unidade (exceto entre os valores 0 e 1,0), baseado na avaliação dos sistemas funcionais e na capacidade de deambulação do paciente.

A avaliação dos sistemas funcionais determina principalmente a pontuação dos escores de 0 a 4,0. Pontuações acima de 4,5 são muito influenciadas pela capacidade de deambulação do indivíduo, principalmente a capacidade de caminhar certas distâncias e a necessidade de apareIhos de auxílio, como apoio unilateral, bilateral, ou cadeira de rodas.

Avaliação da QVRS - O impacto da EM na QVRS foi avaliada pela comparação dos escores de domínios e compo- 
nentes do SF-36 entre os grupos controle e portadores de $E M$, entre os pacientes com EDSS $\leq 3,5$ e $\geq 4,0$ e entre o grupo controle e portadores com EDSS $\leq 3,5$ para a avaliação do impacto da doença em pacientes com menor incapacidade física. Foram verificadas as correlações entre os escores dos domínios e componentes do SF-36 e tempo de doença, tempo de diagnóstico e presença de sintomas.

Estatística - A análise estatística descritiva foi utilizada para a caracterização sócio-demográfica dos grupos controle e portadores de EM.

O coeficiente de correlação de Pearson foi utilizado para determinar as correlações entre os escores dos domínios e componentes do SF-36 e tempo de doença, tempo de diagnóstico e sintomas.

Uma vez que a distribuição é normal e as amostras são independentes e heterocedásticas, o teste $t$ foi utilizado para comparar as médias das pontuações obtidas pelos portadores de EM com as médias do grupo controle e para comparar os escores obtidos pelos pacientes com EDSS $\leq 3,5$ e $\geq 4,0$.

O tamanho do efeito entre duas amostras para cada domínio foi calculado subtraindo-se a média dos escores do grupo de portadores de EM da média dos escores do grupo controle e dividindo-se o resultado pelo desvio padrão do grupo controle. Também foi medido o tamanho do efeito para avaliar as diferenças entre os escores dos grupos com EDSS $\leq 3,5$ e $\geq 4,0$, e entre os escores do grupo controle e o de portadores com EDSS $\leq 3,5$. O tamanho do efeito foi considerado pequeno entre 0,20 e 0,49 , moderado entre 0,50 e 0,79 e grande acima de 0,80 .

O nível de significância para rejeição da hipótese de nulidade considerado foi $p<0,05$, em uma prova bilateral.

\section{RESULTADOS}

Características populacionais - Foram convidados 26 portadores de EM. Deste grupo, dois se recusaram a participar, e um não conseguiu responder ao questionário por déficit cognitivo, totalizando 23 participantes. Nenhum paciente teve surtos nos três meses precedentes à entrevista. O grupo controle constituiu-se por 69 indivíduos saudáveis, pareados por sexo com o grupo de pacientes.

A maioria dos portadores de EM era do sexo feminino (82,6\%). A média de idade foi 39,48 anos (18 a 60 anos; desvio padrão: 12,3) e 31,55 anos (18 a 56 anos; desvio padrão: 9,7 ) entre os pacientes e o grupo controle, respectivamente. Em ambos os grupos, a distribuição pela raça, estado civil, escolaridade e renda familiar foi semelhante. Entretanto, encontrou-se maior proporção de aposentados e inativos entre os portadores $(70 \%)$ que no grupo controle $(16 \%)$.

O tempo médio de doença e o tempo de diagnóstico dos portadores de EM foram 118,5 meses (desvio padrão: 125,7 meses) e de 52,5 meses (desvio padrão:
Tabela 1. Sinais, sintomas e forma evolutiva da esclerose múltipla no grupo de portadores.

\begin{tabular}{|c|c|c|}
\hline & $\mathrm{N}$ & $\%$ \\
\hline \multicolumn{3}{|l|}{ Manifestação inicial } \\
\hline Piramidal & 9 & 39,1 \\
\hline Sensitivo & 4 & 17,4 \\
\hline Medula & 4 & 17,4 \\
\hline Neurite óptica & 4 & 17,4 \\
\hline Tronco & 2 & 8,7 \\
\hline Crise epiléptica & 1 & 4,3 \\
\hline \multicolumn{3}{|c|}{ Sinais e sintomas no curso da doença } \\
\hline Motor & 20 & 87,0 \\
\hline Sensitivo & 17 & 73,9 \\
\hline Cerebelar & 15 & 65,2 \\
\hline Dor & 15 & 65,2 \\
\hline Fadiga & 14 & 60,9 \\
\hline Alterações esfincterianas & 13 & 56,5 \\
\hline Sintomas depressivos & 12 & 52,2 \\
\hline Neurite óptica & 11 & 47,8 \\
\hline Tronco encefálico & 11 & 47,8 \\
\hline Intolerância ao calor & 7 & 30,4 \\
\hline Espasticidade & 7 & 30,4 \\
\hline Sintomas paroxísticos & 4 & 17,4 \\
\hline Sinal de Lhermitte & 4 & 17,4 \\
\hline Alterações cognitivas & 4 & 17,4 \\
\hline Sintoma de Uhtoff & 3 & 13,0 \\
\hline \multicolumn{3}{|l|}{ Forma evolutiva } \\
\hline Remitente-recorrente & 20 & 87,0 \\
\hline Secundariamente progressiva & 2 & 8,7 \\
\hline Primariamente progressiva & 1 & 4,3 \\
\hline Progressiva recorrente & 0 & 0 \\
\hline
\end{tabular}

27,0 meses), respectivamente. O EDSS variou entre 0 e 7,0 com média de 3,96 e mediana de 4,0; doze pacientes $(52,2 \%)$ apresentaram alguma incapacidade ambulatorial (EDSS $\geq 4,0$ ). As demais características clínicas estão listadas na Tabela 1.

Avaliação da qualidade de vida - Em todos os domínios e componentes estudados, observou-se que os portadores de EM apresentaram escores significativamente menores que os controles $(p<0,05)$. $O$ tamanho do efeito é grande em todos os domínios e componentes do SF-36, e foi nitidamente maior no componente físico em comparação ao mental (Tabela 2).

Os escores obtidos pelos portadores de EM com EDSS $\leq 3,5$ e $\geq 4,0$ são demonstrados na Tabela 3 . 0 grupo com maior incapacidade segundo o EDSS apresentou escores significativamente menores nos domínios capacidade funcional, aspectos físicos, dor, estado geral da saúde e vitalidade, e no componente físico. O tamanho do efeito foi grande em todos es- 
Tabela 2. Distribuição das médias, desvios padrão, diferenças e tamanho do efeito dos escores dos domínios e componentes do SF36 nos portadores de EM e no grupo controle.

\begin{tabular}{|c|c|c|c|c|c|c|}
\hline \multirow{2}{*}{$\begin{array}{l}\text { Domínios e } \\
\text { componentes }\end{array}$} & \multicolumn{2}{|c|}{ Controles } & \multicolumn{2}{|c|}{ Portadores } & \multirow{2}{*}{$\begin{array}{c}\text { Diferença } \\
\text { entre médias }\end{array}$} & \multirow{2}{*}{$\begin{array}{l}\text { Tamanho } \\
\text { do efeito }\end{array}$} \\
\hline & Média & Desvio padrão & Média & Desvio padrão & & \\
\hline Capacidade funcional & 94,9 & 8,0 & 43,3 & 30,2 & 51,7 & 6,5 \\
\hline Aspectos físicos & 94,6 & 14,1 & 44,6 & 43,9 & 50,0 & 3,6 \\
\hline Dor & 87,8 & 12,5 & 55,3 & 32,5 & 32,5 & 2,6 \\
\hline Estado geral da saúde & 87,6 & 10,9 & 56,3 & 25,1 & 31,3 & 2,9 \\
\hline Aspectos emocionais & 88,4 & 26,1 & 50,7 & 43,7 & 37,7 & 1,4 \\
\hline Vitalidade & 78,0 & 14,6 & 53,5 & 26,6 & 24,6 & 1,7 \\
\hline Saúde mental & 78,7 & 15,7 & 58,6 & 24,3 & 20,1 & 1,3 \\
\hline Aspectos sociais & 89,7 & 15,5 & 64,1 & 32,9 & 25,5 & 1,6 \\
\hline Componente físico & 92,3 & 6,0 & 47,8 & 26,1 & 44,5 & 7,4 \\
\hline Componente mental & 82,0 & 14,8 & 54,0 & 26,2 & 28,0 & 1,9 \\
\hline
\end{tabular}

Tabela 3. Escores dos domínios e componentes do SF-36 obtidos pelos portadores de EM com EDSS $\leq 3,5$ e $\geq 4,0$.

\begin{tabular}{|c|c|c|c|c|c|c|}
\hline \multirow{2}{*}{$\begin{array}{l}\text { Domínios e } \\
\text { componentes }\end{array}$} & \multicolumn{2}{|c|}{ Portadores EDSS $\leq 3,5$} & \multicolumn{2}{|c|}{ Portadores EDSS $\geq 4,0$} & \multirow{2}{*}{$\begin{array}{c}\text { Diferença } \\
\text { entre médias }\end{array}$} & \multirow{2}{*}{$\begin{array}{l}\text { Tamanho } \\
\text { do efeito }\end{array}$} \\
\hline & Média & Desvio padrão & Média & Desvio padrão & & \\
\hline Capacidade funcional & 60,4 & 26,3 & 27,5 & 25,0 & $32,9 *$ & 1,2 \\
\hline Aspectos físicos & 59,1 & 40,7 & 31,3 & 44,1 & $27,8^{*}$ & 0,7 \\
\hline Dor & 70,2 & 18,6 & 41,7 & 37,1 & $28,5^{*}$ & 1,5 \\
\hline Estado geral da saúde & 67,3 & 23,0 & 46,3 & 24,0 & $21,0 *$ & 0,9 \\
\hline Aspectos emocionais & 45,4 & 40,2 & 55,5 & 47,8 & $-10,1$ & - \\
\hline Vitalidade & 67,3 & 20,4 & 40,8 & 26,0 & $26,5^{*}$ & 1,3 \\
\hline Saúde mental & 66,6 & 19,9 & 51,3 & 26,6 & 15,3 & - \\
\hline Aspectos sociais & 71,6 & 26,3 & 57,3 & 37,9 & 14,3 & - \\
\hline Componente físico & 62,7 & 22,9 & 34,0 & 21,4 & $28,7^{*}$ & 1,2 \\
\hline Componente mental & 60,2 & 22,4 & 48,3 & 29,0 & 11,9 & - \\
\hline
\end{tabular}

${ }^{*} p<0,05$

Tabela 4. Distribuição das médias, desvios padrão, diferenças e tamanho do efeito dos escores dos domínios e componentes do SF-36 nos portadores de EM com EDSS $\leq 3,5$ e no grupo controle.

\begin{tabular}{|c|c|c|c|c|c|c|}
\hline \multirow{2}{*}{$\begin{array}{l}\text { Domínios e } \\
\text { componentes }\end{array}$} & \multicolumn{2}{|c|}{ Controles } & \multicolumn{2}{|c|}{ Portadores EDSS $\leq 3,5$} & \multirow{2}{*}{$\begin{array}{c}\text { Diferença } \\
\text { entre médias }\end{array}$} & \multirow{2}{*}{$\begin{array}{l}\text { Tamanho } \\
\text { do efeito }\end{array}$} \\
\hline & Média & Desvio padrão & Média & Desvio padrão & & \\
\hline Capacidade funcional & 94,9 & 8,0 & 60,5 & 26,3 & 34,4 & 4,3 \\
\hline Aspectos físicos & 94,6 & 14,1 & 59,1 & 40,7 & 35,5 & 2,5 \\
\hline Dor & 87,8 & 12,5 & 70,2 & 18,6 & 17,6 & 1,4 \\
\hline Estado geral da saúde & 87,6 & 10,9 & 67,3 & 22,3 & 20,3 & 1,9 \\
\hline Aspectos emocionais & 88,4 & 26,1 & 45,4 & 40,2 & 43,0 & 1,9 \\
\hline Vitalidade & 78,0 & 14,6 & 67,3 & 20,4 & 10,7 & 0,7 \\
\hline Saúde mental & 78,7 & 15,7 & 66,5 & 19,9 & 12,2 & 0,8 \\
\hline Aspectos sociais & 89,7 & 15,5 & 71,6 & 26,3 & 18,1 & 1,2 \\
\hline Componente físico & 92,3 & 6,0 & 62,8 & 22,8 & 29,5 & 4,9 \\
\hline Componente mental & 82,0 & 14,8 & 60,3 & 22,4 & 21,7 & 1,5 \\
\hline
\end{tabular}


ses domínios e componente, exceto no domínio aspectos físicos, cujo tamanho do efeito foi moderado. Não houve diferença significativa no componente mental.

Em relação ao grupo controle, nos portadores com EDSS $\leq 3,5$ a pontuação foi significativamente menor em todos os domínios e componentes do SF36; todas as diferenças apresentaram tamanho do efeito grande, com exceção do domínio vitalidade, com tamanho do efeito moderado (Tabela 4).

Não foram observadas correlações entre os domínios e componentes do SF-36 com tempo de doença, tempo de diagnóstico e sintomas iniciais. Entre os sintomas relatados no decorrer da doença, os que se correlacionaram mais fortemente e com o maior número de domínios do SF-36 foram sintomas depressivos e intolerância ao calor. A intolerância ao calor se correlacionou com os domínios aspectos físicos $(r=-0,58 ; p=0,004)$, estado geral da saúde $(r=$ $-0,48 ; p=0,02)$, aspectos emocionais $(r=-0,51 ; p=0,01)$, vitalidade $(r=-0,42, p=0,04)$ e aspectos sociais $(r=-0,42$; $p=0,04)$, e com o componente mental $(r=-0,49 ; p=0,02)$. Observou-se correlação entre presença de sintomas depressivos e escores dos domínios capacidade funcional $(r=-0,58 ; p=0,003)$, estado geral da saúde $(r=$ $-0,45 ; p=0,03)$, vitalidade $(r=-0,42 ; p=0,04)$ e saúde mental $(r=-0,42 ; p=0,04)$.

\section{DISCUSSÃO}

O presente estudo auxilia a revelar como os portadores de EM percebem o impacto da doença em suas vidas. Este é o primeiro estudo realizado no Brasil que compara a QVRS em portadores de EM com a de uma população saudável. A utilização de um instrumento genérico de avaliação de QVRS - a versão brasileira do SF-36 - e a comparação com um grupo controle possibilitaram confirmar a hipótese de que os portadores de EM apresentam prejuízo na QVRS nos domínios físicos e psicossociais, em relação à população saudável. De acordo com o tamanho do efeito, percebe-se que esse impacto é importante em todos os domínios, mas é nitidamente mais pronunciado no construto físico.

Em vários estudos de QVRS na EM, a comparação do desempenho obtido pelos pacientes em relação à população normativa mostrou resultados consistentes com um impacto geral negativo causado pela doença em todos os domínios ${ }^{10,16,17}$; entretanto, em alguns estudos, não foram encontradas diferenças significativas em um ou mais domínios ${ }^{18,19}$. Apesar de algumas diferenças pontuais entre si, todos estes estudos mostram que os domínios de função física apresentam maior prejuízo que os domínios psicossociais. Esse achado é consistente com os parâmetros clínicos que demonstram a limitação motora.

O impacto negativo causado pela EM pode ser conseqüência de vários fatores, dentre os quais a incapacidade causada pela doença seria apenas um deles. O fato de se descobrir portador de uma doença neurológica crônica, evolutiva, de curso imprevisível, gradualmente incapacitante e até o momento sem cura, pode repercutir de forma contundente na vida dos pacientes, mesmo nas fases iniciais ${ }^{20}$.

Uma vez que as manifestações clínicas da EM são heterogêneas, comparações entre grupos de pacientes conforme o nível de incapacidade física podem fornecer informações relevantes sobre particularidades da repercussão da doença na QVRS. No presente estudo, os portadores de EM foram divididos utilizando-se como marco a fase em que se inicia a manifestação de incapacidade ambulatorial (EDSS 4,0 ). Pacientes sem incapacidade à marcha (EDSS $\leq 3,5)$ apresentam escores piores em todos os domínios quando comparados ao grupo controle, e, da mesma forma que no grupo de portadores como um todo, a repercussão foi maior nos domínios de função física. Além disso, verificou-se que a doença provoca maior impacto nos domínios físicos em portadores com maior grau de comprometimento motor (EDSS $\geq 4,0$ ) em relação àqueles menos comprometidos (EDSS $\leq 3,5$ ); entretanto, nos domínios mentais e sociais, ambos os grupos apresentam desempenho semelhante.

Em geral, os estudos que avaliaram a QVRS entre grupos divididos de acordo com a incapacidade ao EDSS mostraram que, quanto maior o comprometimento, maior o impacto nos domínios físicos ${ }^{17,20,21}$. Alguns desses estudos mostram que há também maior impacto nos domínios mentais com a progressão da incapacidade física ${ }^{17,20}$. Esse fato pode ser explicado, ao menos em parte, pela forma não padronizada de divisão desses grupos nos diversos estudos, com a utilização de diferentes níveis do EDSS como marco divisório.

A piora da função física percebida pelos pacientes não acompanhada pela percepção de deterioração das funções mentais com a progressão do EDSS - conforme visto no presente estudo - pode ser explicada por um processo de acomodação psicológica do portador à doença (resiliência). Por outro lado, essa estabilidade relativa dos domínios mentais pode ser devida a características próprias do instrumento. Um estudo sugere que o questionário SF-36 aparen- 
temente superestima a saúde mental em portadores de $\mathrm{EM}^{18}$. Esses fatos necessitam de melhor esclarecimento através de novos estudos.

Sintomas que acompanham a doença como fadiga, dores, disfunção sexual e esfincteriana, déficit cognitivo, e comorbidades como a depressão, também podem interferir significativamente na QVRS em portadores de $\mathrm{EM}^{20,22-24}$. No presente estudo, encontramos correlações significativas entre sintomas depressivos e intolerância ao calor com domínios físicos e mentais do SF-36. Esta última, em particular, ainda não foi estudada em outros trabalhos. Uma explicação possível para esse fato seria a exposição freqüente desses pacientes a temperaturas elevadas durante boa parte do ano, o que é próprio da região estudada. São necessários outros estudos para se verificar se esses achados também ocorrem em populações expostas a climas mais amenos.

Os portadores de EM no presente estudo são, em sua maioria, inativos e aposentados, ao contrário do grupo controle. Isso se deve principalmente à incapacidade promovida pela doença que, por acometer principalmente adultos jovens, leva a importante prejuízo na fase mais produtiva de suas vidas.

Os resultados desse estudo precisam ser avaliados à luz de algumas críticas. Por exemplo, o tamanho da amostra recrutada é pequeno. Ainda assim, a população avaliada corresponde aproximadamente a um terço do número de portadores de EM esperado para o município de Uberlândia, de acordo com estudos de prevalência realizados em outros municípios da região sudeste do $\mathrm{Brasi}^{25-27}$. O perfil demográfico e clínico é semelhante ao encontrado em outros estudos populacionais da região sudeste do Brasil. A proporção de mulheres acometidas é maior que a verificada em estudos realizados em outros países ${ }^{1}$ e pode refletir uma característica da doença e/ou da população brasileira ou, pelo menos, da região sudeste do Brasil. A distribuição dos tipos de evolução clínica é semelhante à encontrada na literatura ${ }^{1}$; entretanto, o baixo número de portadores com as formas progressivas da doença não permitiu a comparação da QVRS dos pacientes com as diferentes formas de evolução.

Por se tratar de um instrumento genérico, o SF-36 não aborda de forma específica vários sintomas comuns em portadores de EM, como alterações esfincterianas, disfunções sexuais e problemas cognitivos. Dessa forma, sua utilização para a avaliação de intervenções pode ser limitada. Por outro lado, possibilita melhor avaliação do impacto da doença na QVRS de portadores de EM, permitindo comparações com a população geral ou com grupos de pacientes com outras situações crônicas.

Os valores dos desvios padrão dos escores dos domínios do SF-36 foram elevados no presente estudo. Isso poderia ser considerado como conseqüência do tamanho da amostra. Entretanto, estudos realizados com amostras maiores mostraram desvios padrão semelhantes ${ }^{11,16,18,19}$. Dessa forma, esse fenômeno deve acontecer, principalmente, pela heterogeneidade das manifestações clínicas da doença e da percepção do impacto da EM na vida dos portadores.

Concluindo, portadores de EM apresentam um importante impacto negativo em todas as dimensões da QVRS, com maior comprometimento nos domínios de função física. Esses resultados reforçam a necessidade de uma equipe interdisciplinar no acompanhamento do paciente, com especial cuidado para os aspectos físicos, psicológicos e sociais. Os investimentos em programas de saúde devem estar atentos quanto aos prejuízos causados pela doença para disponibilizar, de modo planejado, os recursos apropriados a cada nível de envolvimento. Novos estudos são necessários para a melhor avaliação do impacto da EM em portadores brasileiros com a utilização conjunta de instrumentos genéricos e específicos para a determinação mais detalhada da influência dos sintomas, e para a verificação da interferência das intervenções medicamentosas e não medicamentosas na QVRS percebida pelos pacientes. Por se tratar de doença pouco prevalente, a promoção de estudos multicêntricos com maior número de pacientes pode originar resultados mais consistentes.

\section{REFERÊNCIAS}

1. O'Connor P. Key issues in the diagnosis and treatment of multiple sclerosis: an overview. Neurology 2002;59(Suppl 3):S1-S33.

2. Paty DW, Ebers GC. Clinical features. In Paty DW, Ebers GC (Eds). Multiple sclerosis. Philadelphia: FA Davis, 1997:135-191.

3. Guyatt GH, Naylor CD, Juniper E, Heyland DK, Jaeschke R, Cook DJ Users' guides to the medical literature: XII. How to use articles about health-related quality of life: evidence-based medicine working group. JAMA 1997;277:1232-1237.

4. WHOQOL Group. The World Health Organization Quality Of Life Assessment (WHOQOL): position paper from the World Health Organization. Soc Sci Med 1995;41:1403-1409.

5. Theunissen NC, Vogels TG, Koopman HM, et al. The proxy problem child report versus parent report in health-related quality of life research. Qual Life Res 1998; 7:387-397.

6. Ware JE, Kosinski M, Gandek B. SF-36 Health survey: manual and Interpretation guide. Boston (MA): The Health Institute, New England Medical Center, 1993.

7. Ciconelli RM, Ferraz MB, Santos W, Meinão I, Quaresma MR. Tradução para a língua portuguesa e validação do questionário genérico de avaliação de qualidade de vida SF-36 (Brasil SF-36). Rev Bras Reumatol 1999;39:143-150.

8. Nortvedt MW, Riise T, Myhr KM, Nyland HI. Quality of life in multiple sclerosis: measuring the disease effects more broadly. Neurology 2000;53:1091-1103. 
9. Freeman JA, Hobart JC, Langdon DW, Thompson AJ. Clinical appropriateness: a key factor in outcome measure selection: The 36-Item Short Form Health Survey in multiple sclerosis. J Neurol Neurosurg Psychiatry 2000;68:150-156.

10. Solari A. Role of health-related quality of life measures in the routine care of people with multiple sclerosis. Health Qual Life Outcomes 2005; 3. Disponível em: http:/ / www.hqlo.com/content/3/1/16

11. Mendes MF, Balsimelli S, Stangehaus G, Tilbery CP. Validação de Escala de Determinação Funcional da Qualidade de Vida na esclerose múltipla para a língua portuguesa. Arq Neuropsiquiatr 2004;62:108-113.

12. Cella DF, Dineen K, Arnason B, et al. Validation of the functional assessment of multiple sclerosis quality of life instrument. Neurology 1996; 47:129-139.

13. McDonald WI, Compston A, Edan G, et al. Recommended diagnostic criteria for multiple sclerosis: guidelines from the International Panel on the Diagnosis of Multiple Sclerosis. Ann Neurol 2001;50:121-127.

14. Morales RR. Avaliação transversal da qualidade de vida em portadores de esclerose múltipla por meio de um instrumento genérico (SF-36). 2005. 96f. Dissertação. Uberlândia, 2005.

15. Kurtzke JF. Rating neurological impairment in multiple sclerosis: an Expanded Disability Status Scale (EDSS). Neurology 1983;33:1444-1452.

16. Pfennings L, Cohen L, Miller D, et al. Using the Short Form-36 with multiple sclerosis patients in five countries: a cross-cultural comparison. Psychol Rep 1999;85:19-31.

17. Canadian Burden of Illness Study Group. Burden of illness of multiple sclerosis: Part II. Quality of life. Can J Neurol Sci 1998;25:31-38.

18. Vickrey BG, Hays RD, Harooni R, Myers LW, Ellison GW. A health-re- lated quality-of-life measure for multiple sclerosis. Qual Life Res 1995; 4:187-206.

19. Pittock SJ, Mayr WT, McClelland RL, et al. Quality of life is favorable for most patients with multiple sclerosis: a population-based cohort study. Arch Neurol 2004;61:679-686.

20. Janssens AC, van Doorn PA, de Boer JB, et al. Anxiety and depression influence the relation between disability status and quality of life in multiple sclerosis. Mult Scler 2003;9:397-403.

21. Nortvedt MW, Riise T, Myhr KM, Nyland HI, Hanestad BR. Type I interferons and the quality of life of multiple sclerosis patients: results from a clinical trial on interferon alfa-2a. Mult Scler 1999;5:317-322.

22. Amato MP, Ponziani G, Rossi F, Liedl CL, Stefanile C, Rossi L. Quality of life in multiple sclerosis: the impact of depression, fatigue and disability. Mult Scler 2001;7:340-344.

23. Nortvedt MW, Riise T, Myhr KM, Landtblom AM, Bakke A, Nyland HI. Reduced quality of life among multiple sclerosis patients with sexual disturbance and bladder dysfunction. Mult Scler 2001;7:231-235.

24. Cutajar R, Ferriani E, Scandellari C, et al. Cognitive function and quality of life in multiple sclerosis patients. J Neurovirol 2000;6(Suppl 2): S186-S190.

25. Callegaro D, de Lolio CA, Radvany J, Tilbery CP, Mendonça RA, Melo AC. The prevalence of multiple sclerosis in the city of São Paulo, Brazil, 1997. Acta Neurol Scand 2001;104:208-213.

26. Rocha FCG, Herrera LCL, Morales RR. Multiple sclerosis in Botucatu, Brazil: a population study. Mult Scler 2002;8(Suppl 1):S41.

27. Lana-Peixoto MA. The prevalence of MS in Belo Horizonte, Brazil. Mult Scler 2002;8(Suppl 1):S38. 